\title{
Active Surveillance in the investigation and analysis of RVF in Western and Central Uganda
}

\author{
Mary L. Nanfuka1, 2, Milton Bahati ${ }^{1,}$ 2, Eugene Arinaitwe ${ }^{1,2}$ \\ ${ }^{1}$ Animal Health, Ministry of Agriculture Animal Industry and Fisheries, Entebbe, Entebbe, Uganda, ${ }^{2}$ National Animal Disease Diagnostics and \\ Epidemiology Center (NADDEC), Entebbe, Entebbe, Uganda \\ Objective
}

To detect presence of circulating Rift Valley Fever virus (RVFv) in animals of Western and Central Uganda following its confirmation in humans. To establish and communicate reliable information using the one health plat form

Significance:

Although in E. Africa RVF was initially detected and known to be a disease endemic in Kenya, the people in Uganda were still hesitating whether the disease is already in existence. Following its first detection in 2016 in Humans there was need to carry out an investigation in the hot spot areas of the human infection to get the real picture and to inform the policy makers for informed decisions.

Introduction

Rift Valley fiver is viral zoonotic disease which was investigated and reported in Uganda in 2010 [1]. For some time now people are not aware whether the disease was still circulating or emerged in animals reared as a result of the inter country trade by the community of the cattle corridor in Uganda, since the last reports in 1968 [2]. The increase in the number of disease outbreaks in some parts of central and western Uganda from 2016 to date and the number of human patients investigated, diagnosed and confirmed with RVF by Ministry Of Health $(\mathrm{MOH})$ under the one health program, has placed the disease to be among the top reemerging diseases in the country[3,4] and number 5 of the Multisectoral prioritization of zoonotic diseases in Uganda, 2017 under One Health perspective [5].

\section{Methods}

Rift valley Fever was investigated in cattle, goats and sheep of Gomba,Mityana, Kiboga and Kiruhura in Central and Western Uganda. This followed 2 people that had been confirmed with RVF in 2016 [1] Samples were aseptically collected from hot places from 543 victim's animals including those of the neighbouring areas covering the victims routes of movement plus those areas where people were still sick and where death had reportedly occurred. Samples were then delivered to NADDEC laboratory from where tests were conducted.

\section{Results}

Samples were screened using a competition IgG ELISA, then IgM ELISA to capture the recently infected animals. The positive samples from the IgM ELISA were then confirmed using RT-PCR; 169/543 (31\%) tested positive to IgG screening ELISA indicating exposure to RVF. The actual infection was found to be 13\% (22/169) with IgM ELISA and 3/22 (13.6\%) with RT-PCR.

\section{Conclusions}

Zoonotic diseases continue to be a public health burden to the people of Uganda. Considering some people's behavior of eating the sick and dead animals, has posed a difficult situation to combat the ailment which has resulted in negative socioeconomic impacts, affecting the national policies that range from health security to control of diseases. Uganda has however developed capacity to investigate, test and confirm RVF disease. Since exposure was found in all animal species, detailed active surveillance plan and procedures have been set up to investigate any additional cases in animals to reduce chances of spread to humans and to cub international spread and also to determine the magnitude of exposure.

\section{Acknowledgement}

I acknowledge my coauthors for the knowledge and skills added to this manuscript and the entire staff of the National Animal Disease Diagnostics and Epidemiology Center for all the support granted to me in this work 


\section{References}

1. Nabukenya I, et al. 2016. Investigation and response to Rift Valley Fever and Yellow Fever outbreaks in humans in Uganda. Int J Infect Dis. 53, 129.

2. Nyakarahuka L, et al. 2018. Prevalence and risk factors of Rift valley in humans and animals from kabale, 2016. PLoS Negl Trop Dis. 12(5). doi:10.1371/journal.pntd.0006412. PubMed

3. Wang LF, Crameri G. Emerging zoonotic viral diseases.Rev Sci Tech Int Epiz.2014;33

4. Institute of Medicine (U.S.), Committee on Achieving Sustainable Global Capacity for surveillance and Response to Emerging Diseases of Zoonotic Origin, Keusch G. Sustaining global surveillance and response to emerging zoonotic diseases, 2009

5. Munyua P, Bitek A, Osoro E, Pieracci EG, Muema J, et al. 2016. Prioritization of Zoonotic Diseases in Kenya,2015 [PubMed]. PLoS One. 11, e0161576. doi:10.1371/journal.pone.0161576. PubMed

6. Sekamatte M, et al. 2018. Multisectoral prioritization of zoonotic diseases in Uganda, 2017: A One Health perspective. PLoS One. 13(5), e0196799. PubMed

Table showing RVF positivity in the different animal species using ELISA and RT-PCR

\begin{tabular}{|c|c|c|c|c|c|c|c|}
\hline District & Goats & Cattle & Sheep & +IgG ELISA & +IgM ELISA & RT-PCR & Negative \\
\hline Gomba & 0 & 114 & 0 & 27 & 7 & 0 & 80 \\
\hline Mityana & 46 & 76 & 18 & 45 & 6 & 0 & 89 \\
\hline Kiboga & 20 & 80 & 51 & 40 & 4 & 1 & 105 \\
\hline Kiruhura & 55 & 63 & 20 & 57 & 5 & 75 \\
\hline
\end{tabular}

121 Goats, 333 cattle and 89 sheep, 169 tested positive, 22 tested positive and 3 were positive with RT-PCR. 349 tested Negative. A total of 543 samples were collected for analysis 